\title{
Actualización en la interpretación de la medición del pH e impedanciometría
}

\section{Updated interpretation of Impedance-pH monitoring}

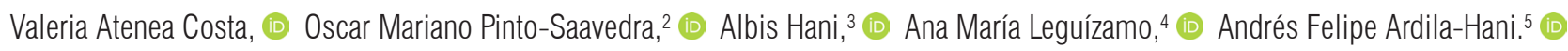

\author{
GacCeso abierto \\ Citación: \\ Costa V, Pinto-Saavedra 0, Hani A, \\ Leguízamo AM, Ardila-Hani AF. Actualización \\ en la interpretación de la medición del \\ pH e impedanciometría. Rev Colomb \\ Gastroenterol. 2021:36(1):73-80. https://doi. \\ org/10.22516/25007440.608 \\ Médica internista, gastroenteróloga. Hospital \\ Universitario San Ignacio. Bogotá, Colombia. \\ 2 Médico internista, fellow de gastroenterología, \\ Pontificia Universidad Javeriana. Bogotá, Colombia. \\ ${ }^{3}$ Médica internista y gastroenteróloga. Profesora \\ titular, Pontificia Universidad Javeriana. Bogotá, \\ Colombia. \\ ${ }^{4}$ Médica internista y gastroenteróloga. Profesor \\ ad honorem, Pontificia Universidad Javeriana. \\ Bogotá, Colombia. \\ ${ }^{5}$ Médico internista y gastroenterólogo. Profesor ad \\ honorem, Pontificia Universidad Javeriana. Hospital \\ Universitario San Ignacio. Bogotá, Colombia. \\ *Correspondencia: Valeria Atenea Costa. \\ ateneacosta.82@gmail.com \\ Fecha recibido: $\quad 03 / 07 / 20$ \\ Fecha aceptado: 19/11/20
}

\section{Resumen}

La enfermedad por reflujo gastroesofágico (ERGE) se define como el tránsito anormal del contenido gástrico hacia el esófago, que se da por una alteración de la barrera antirreflujo, causando síntomas o complicaciones. Para su correcto diagnóstico y abordaje terapéutico, se requiere de la integración de hallazgos clínicos, endoscópicos y monitorización del pH esofágico en 24 horas con o sin impedanciometría, la cual debe ser realizada con especificaciones técnicas, y su interpretación debe basarse en la mejor evidencia clínica disponible, con el objetivo de tener diagnósticos precisos que permitan tomar las mejores decisiones con los pacientes.

Recientemente, en el Consenso de Lyon se han incorporado nuevas directrices para el diagnóstico de ERGE por monitorización de pH esofágico, las cuales se revisan en este artículo.

\section{Palabras clave}

Monitorización de pH-impedanciometría, enfermedad por reflujo gastroesofágico, inhibidor de la bomba de protones.

\section{Abstract available to obtain accurate diagnoses that allow making the best decisions to the benefit of patients. monitoring, which are reviewed in this paper. \\ Keywords \\ pH-impedance monitoring, Gastroesophageal reflux disease, Proton-pump inhibitor.}

Gastroesophageal reflux disease (GERD) is defined as the abnormal transit of gastric contents into the esophagus. It is caused by an alteration of the anti-reflux barrier, causing multiple symptoms or complications. In order to achieve accurate diagnosis and proper therapeutic approach, integration of clinical findings, endoscopic findings and 24-hour esophageal pH monitoring, with or without impedancometry, is required. These tests must be performed following technical specifications and their interpretation must be based on the best clinical evidence

Recently, the Lyon Consensus incorporated new guidelines for the diagnosis of GERD by esophageal pH

\section{INTRODUCCIÓN}

La enfermedad por reflujo gastroesofágico (ERGE) es definida como el tránsito anormal del contenido gástrico hacia el esófago, que se da por una alteración en la barrera antirreflujo, causando síntomas o complicaciones (1). Tradicionalmente, la ERGE se ha diagnosticado con base en la presentación de síntomas, los cuales se han clasificado en síntomas típicos (pirosis, regurgitación) y síntomas atípicos (dolor torácico, tos, entre otros); y se evalúa la res- 
puesta o no al tratamiento con supresores de ácido, específicamente inhibidores de la bomba de protones (IBP) (2, $3)$. Sin embargo, como parte de los estudios diagnósticos complementarios, además de las manifestaciones clínicas, se hace necesaria la realización de estudios adicionales, como la endoscopia digestiva alta, en la cual es posible documentar hallazgos que confirmen el diagnóstico (esófago de Barrett, estenosis péptica, esofagitis grados C y D, según la clasificación de los Ángeles) (4).

La endoscopia puede ser normal hasta en el $60 \%$ de los en pacientes con ERGE, condición denominada ERGE no erosivo. La monitorización de $\mathrm{pH}$ esofágico con o sin impedanciometría se considera el estudio de elección para confirmar el diagnóstico de ERGE aun con estudios endoscópicos normales, y además permite establecer durante el seguimiento en los pacientes con persistencia sintomática si existe una adecuada respuesta a la terapia supresora de ácido y clasificar el tipo de reflujo (ácido y no ácido); entre sus indicaciones sin terapia supresora con IBP están la valoración prequirúrgica en el paciente con ERGE que se considera candidato a cirugía antirreflujo, confirmación de ERGE, síntomas atípicos, después de la realización de cirugía antirreflujo y los síntomas persistentes a pesar del tratamiento con IBP; se debe realizar con terapia de IBP en pacientes con esofagitis grados $\mathrm{C}$ y D de los Ángeles, esófago de Barrett, estenosis péptica, o $\mathrm{pH}$-impedanciometría previa positiva $(5,6)$.

\section{EPIDEMIOLOGÍA}

La prevalencia mundial estimada de la ERGE es del $8 \%$ al $33 \%$, incluye todos los grupos de edad y ambos sexos; además, constituye un costo alto en salud, dado el uso frecuente de los IBP, los cuales son de venta libre, así como las pruebas diagnósticas indicadas con alta frecuencia (5, 7). En el estudio realizado en Colombia que se llevó a cabo en 4 ciudades capitales aplicando el cuestionario GERDQ (gastroesophageal reflux disease questionnaire) se estableció que la prevalencia de síntomas de reflujo es de $11,98 \%$, y se encontró una asociación con comorbilidades como la hipertensión arterial (8).

\section{INDICACIONES}

La pH-impedanciometría es útil para confirmar el diagnóstico de ERGE en pacientes con esofagogastroduodenoscopia normal, síntomas atípicos, cuando se contempla la realización de cirugía antirreflujo y en el contexto de refractariedad al tratamiento con los IBP. La pH-impedanciometría se indica sin terapia supresora de ácido cuando la ERGE no ha sido comprobada, no hay estudio de monitorización de la medición del $\mathrm{pH}$ previa y también en pacientes en plan de cirugía antirreflujo (esto con el fin de no ofrecer un tratamiento quirúrgico a un paciente que no tenga la enfermedad); sin embargo, en pacientes con ERGE comprobada (esófago de Barrett, estenosis péptica, esofagitis grados $\mathrm{C}$ y $\mathrm{D}$, según la clasificación de los Ángeles) o un estudio de monitorización de $\mathrm{pH}$ previo positivo se recomienda la evaluación se haga con terapia supresora de ácido a doble dosis, para establecer una asociación entre los síntomas refractarios y los episodios de reflujo. La impedanciometría da un valor agregado a la monitorización de la medición del $\mathrm{pH}$, pues permite establecer reflujos de tipo no ácido, dado que la mayoría de los episodios de reflujo en pacientes que reciben supresión con IBP son débilmente ácidos. En la Figura 1 se ilustran las indicaciones de $\mathrm{pH}$-impedanciometría con y sin tratamiento supresor de ácido (9).

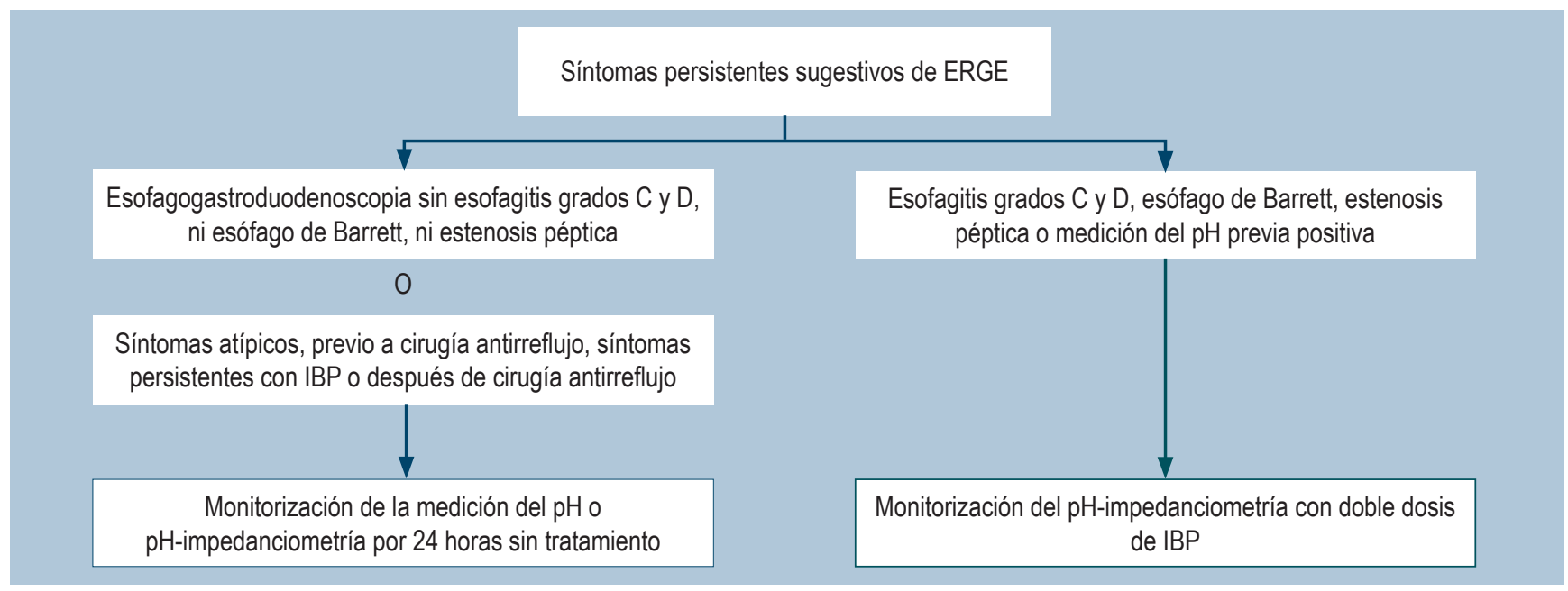

Figura 1. Indicaciones de $\mathrm{pH}$-impedanciometría con y sin tratamiento (9). Tomado de: Roman S et al. Neurogastroenterol Motil. 2017;29(10):1-15. 


\section{ASPECTOS TÉCNICOS DE LA PRUEBA}

Para la realización de la $\mathrm{pH}$-impedanciometría sin terapia supresora de ácido se recomienda suspender 7 días antes el IBP; 3 días antes, los antagonistas $\mathrm{H}_{2}$; 6 a 12 horas antes, los antiácidos; y 5 a 7 días antes, los procinéticos. Previo a la colocación del catéter transnasal para la monitorización de $\mathrm{pH}$-impedanciometría, el paciente debe tener ayuno de 6 horas para evitar la emesis y broncoaspiración. Durante la monitorización se les debe indicar a los pacientes que deben consumir sus comidas habituales, además de que deben llevar registro de la ingesta, sus períodos en posición vertical y supino (registrando el momento de acostarse), y el registro de los síntomas $(9,10)$.

El catéter de $\mathrm{pH}$-impedanciometría consta un sensor de pH y 8 sensores de impedanciometría. La impedanciometría multicanal usa propiedades conductoras inherentes al bolo intraluminal (líquido, gaseoso o mixto), para examinar la presencia y el tránsito del bolo en la luz esofágica. Se dispone de dispositivos que permiten la realización de monitorización combinada (monitorización de impedanciometría y $\mathrm{pH}$ esofágico o impedanciometría y manometría esofágica) $(10,11)$ (Figura 2).

La impedanciometría es una medida de la resistencia total al flujo de corriente alterna, que depende de las propiedades del material en contacto con los electrodos y refleja la presencia de contenido esofágico durante la evaluación $(10,11)$.

Con la impedanciometría es posible diferenciar los bolos líquidos, gaseosos o mixtos. La impedancia permite evaluar

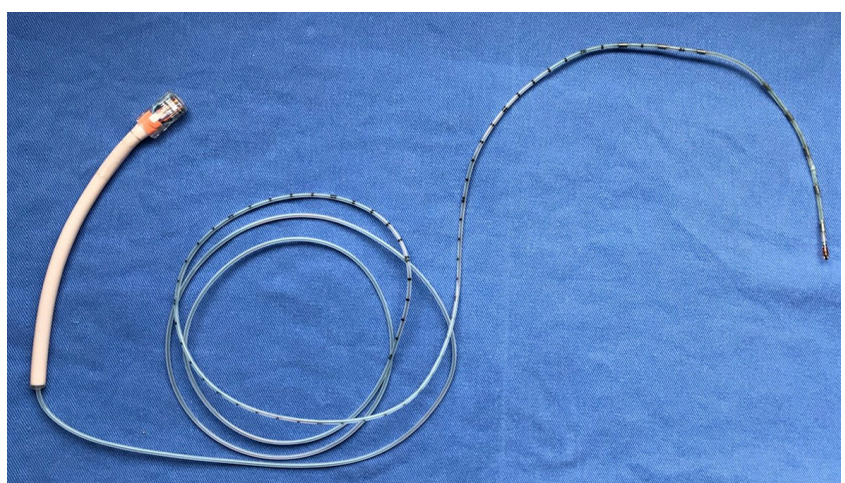

Figura 2. Catéter de $\mathrm{pH}$-impedanciometría (un sensor de $\mathrm{pH}$ y 8 sensores de impedanciometría) Fuente: propiedad de los autores.

el tránsito del bolo esofágico. La dirección del movimiento del bolo se determina en función de la secuencia del tiempo de entrada y salida del bolo a través de diferentes segmentos de medición $(10,11)$ (Figura 3 ).

La prueba de impedancia generalmente se usa en combinación con la monitorización de $\mathrm{pH}$, debido a que aporta información complementaria y permite una mejor evaluación de la ERGE. La impedanciometría combinada con la monitorización de $\mathrm{pH}$ permite la detección de reflujo gastroesofágico de diversas consistencias (ácidos, no ácidos, eventos líquidos y no líquidos); estos hallazgos son relevantes porque en un porcentaje importante $(45 \%)$ de pacientes con ERGE que no responden a terapia supresora de ácido tiene reflujo no ácido, el cual se logra diagnosticar gracias a la impedanciometría $(10,11)$.

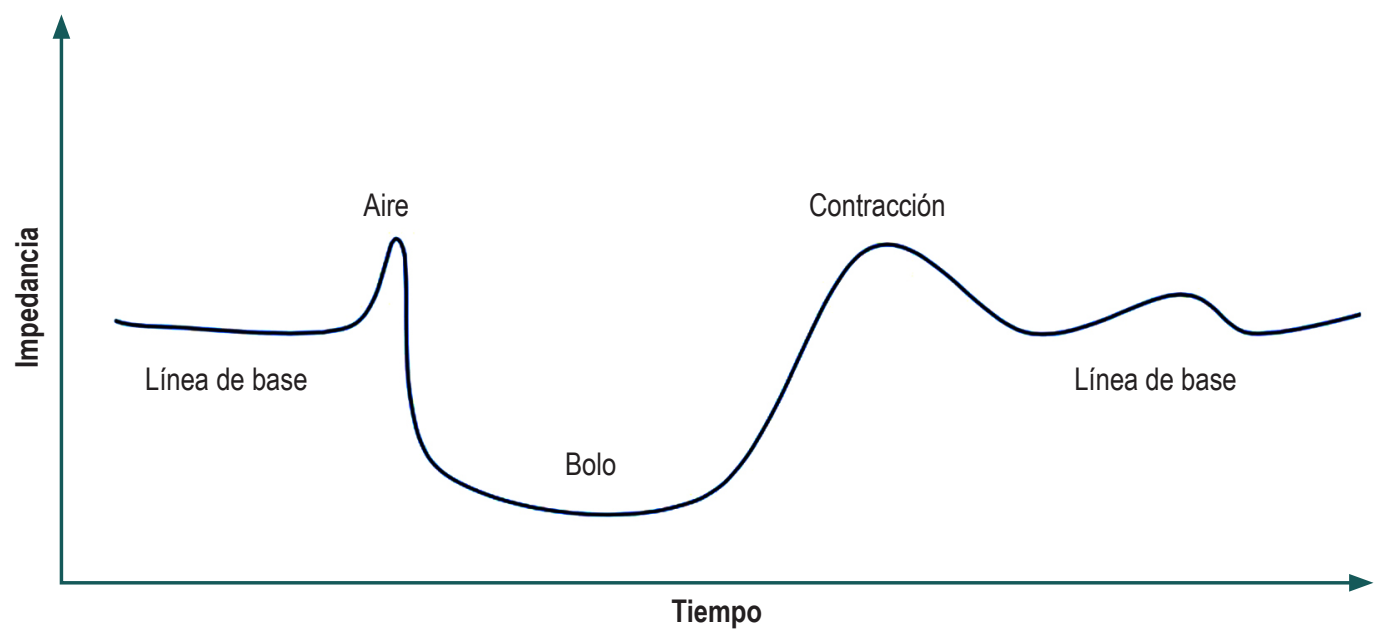

Figura 3. Representación del bolo entre 2 electrodos de impedancia, hecho que inicialmente registra un aumento de la impedancia por el aire que abre paso al bolo en el esófago, con un posterior descenso rápido de la impedancia cuando el bolo se ubica entre los dos electrodos; luego, se registra un aumento de la impedancia que corresponde a la contracción esofágica generada por el impulso del bolo, con un posterior retorno progresivo a la línea de base de la impedancia a medida que el bolo va siendo aclarado del segmento entre los 2 electrodos (10). 
El sensor de $\mathrm{pH}$ se posiciona a $5 \mathrm{~cm}$ por encima del esfínter esofágico inferior, lugar donde no se desplaza el catéter con las degluciones hacia el estómago. Si se ubica a mayor distancia, se ha demostrado un subregistro en los episodios de reflujo. La ubicación del catéter idealmente se debe hacer guiado por manometría esofágica; de esta manera se logra establecer la posición del esfinter esofágico inferior (EEI), para realizar su adecuado posicionamiento. Si no se dispone de esta herramienta, se ubica verificando que el $\mathrm{pH}$ sea alcalino, lo que permite establecer su ubicación en el esófago $5 \mathrm{~cm}$ proximal al EEI (10).

El catéter de $\mathrm{pH}$ debe ser adecuadamente calibrado, previa a su colocación en soluciones amortiguadora con un $\mathrm{pH}$ entre 4 y 7 , siguiendo las instrucciones del fabricante.

\section{ANÁLISIS E INTERPRETACIÓN DE LA MEDICIÓN DEL PH E IMPEDANCIOMETRÍA}

A lo largo de la historia, el método para la evaluación diagnóstica de la ERGE ha venido siendo modificada de acuerdo con varios consensos. En 2006 el Consenso de Montreal definió la ERGE como una alteración esofágica que se presenta cuando el reflujo del contenido del estómago en el esófago causa síntomas o complicaciones; además, agrupó los pacientes con ERGE, de acuerdo con los síntomas, en síndromes esofágicos o extraesofágicos, abordaje que no discrimina bien otras enfermedades esofágicas y extraesofágicas; situación que cuestiona el diagnóstico de ERGE solo por los síntomas $(12,13)$.

El Consenso de Lyon, el más reciente sobre ERGE, propuso que los síntomas pueden ser no confiables e indicó una evaluación objetiva de los pacientes con sospecha de ERGE con un diagnóstico basado en hallazgos endoscópicos (esofagitis grave, estenosis péptica, esófago de Barrett de segmento largo) y hallazgos de $\mathrm{pH}$-impedanciometría de 24 horas, que define la ERGE cuando el tiempo de exposición al ácido (TEA) es $>6 \%$; razón por la cual es el consenso que aporta la mayoría de los criterios de la interpretación de $\mathrm{pH}$ impedanciometría que se describen a continuación (5). Un ejemplo de trazado de una $\mathrm{pH}$-impedanciometría se muestra en la Figura 4.

El puntaje de DeMeester ha sido utilizado en los últimos 50 años para definir la ERGE. Se trata de un puntaje compuesto que mide la exposición al ácido durante la monitorización de 24 horas de la medición del pH. El reflujo ácido se define siempre que el $\mathrm{pH}$ del esófago medido $5 \mathrm{~cm}$ por encima del borde superior del EEI disminuye a 4 o menos. Los parámetros de DeMeester incluyen el número total de episodios de reflujo, tiempo total de $\mathrm{pH}$ esofágico menor de 4 , tiempo de $\mathrm{pH}$ esofágico en posición vertical menor de 4, $\mathrm{pH}$ del esófago en posición supina menor de 4, número de episodios de reflujo mayor de 5 minutos y episodio de reflujo más largo (13).
Para realizar un adecuado análisis e interpretación de una $\mathrm{pH}$-impedanciometría, se recomienda realizarlo por pasos.

\section{Paso 1: duración de la monitorización de pH- impedanciometría}

Evaluar la duración de la monitorización de pH-impedanciometría, la cual debe tener una duración mínima de 16 horas para poder establecer un adecuado análisis $(5,9)$.

\section{Paso 2: determinar el TEA}

El TEA hace referencia al tiempo en que el esófago se expone a un $\mathrm{pH}$ menor de 4 , y se correlaciona con la presencia de ERGE. Si el TEA es menor de $4 \%$, es considerado normal; si es mayor de $6 \%$ en las 24 horas, se considera anormal y establece el diagnóstico de ERGE; si se documenta entre $4 \%$ y $6 \%$, se califica como no concluyente o zona gris y se requiere de una medida adicional para confirmar el diagnóstico de ERGE (Figura 5) (5, 9, 14).

\section{Paso 3: evaluar el número de episodios de reflujo}

Los episodios de reflujo se clasifican como ácidos y no ácidos (incluye los débilmente ácidos). Un número de episodios de reflujo ácido menor de 40 en 24 horas es considerado normal, mientras que un número mayor de 80 es considerado anormal. Cuando se presentan más de 80 episodios de reflujo ácido se establece diagnóstico de ERGE aun cuando el TEA esté en un rango no concluyente o zona gris (entre $4 \%$ y $6 \%$ ). El número de episodios de reflujo no ácido establece el diagnóstico de enfermedad por reflujo no ácido cuando se presentan más de 27 episodios de este tipo sin terapia supresora de ácido; o más de 44 cuando el examen es realizado en presencia de terapia supresora de ácido. Tanto los episodios de reflujo ácido como los no ácidos deben reportarse si se presentaron en posición vertical, supino o posprandial $(5,9,10,15)$.

\section{Paso 4: establecer la asociación de síntomas con los episodios de reflujo}

La asociación de síntomas con los episodios de reflujo comprende aquellas manifestaciones clínicas de ERGE referidas por el paciente que se presentan hasta 2 minutos antes del registro del episodio de reflujo. Para considerar la asociación de un síntoma con los episodios de reflujo, este debe haber sido reportado 3 veces o más en el registro del paciente. La asociación de síntomas con episodios de reflujo comprende dos evaluaciones: el índice sintomático (IS) y la probabilidad de asociación sintomática (PAS). El IS es el porcentaje de eventos sintomáticos precedido por los episodios de reflujo; 


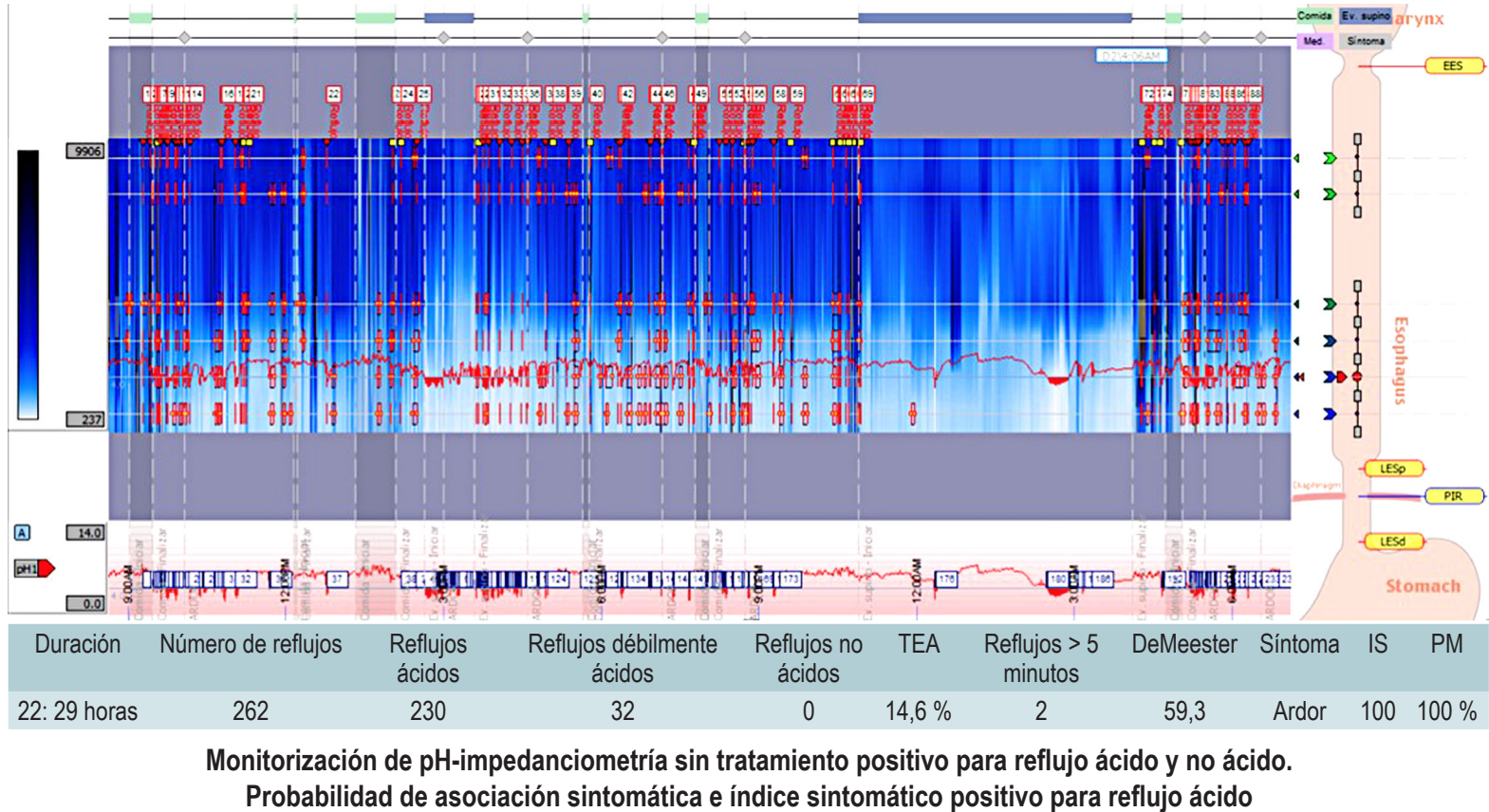

Figura 4. Estudio de monitorización de pH-impedanciometría de 24 horas sin tratamiento. EES: esfinter esofágico superior; PIR: punto de inversión respiratorio. Fuente: propiedad de los autores.

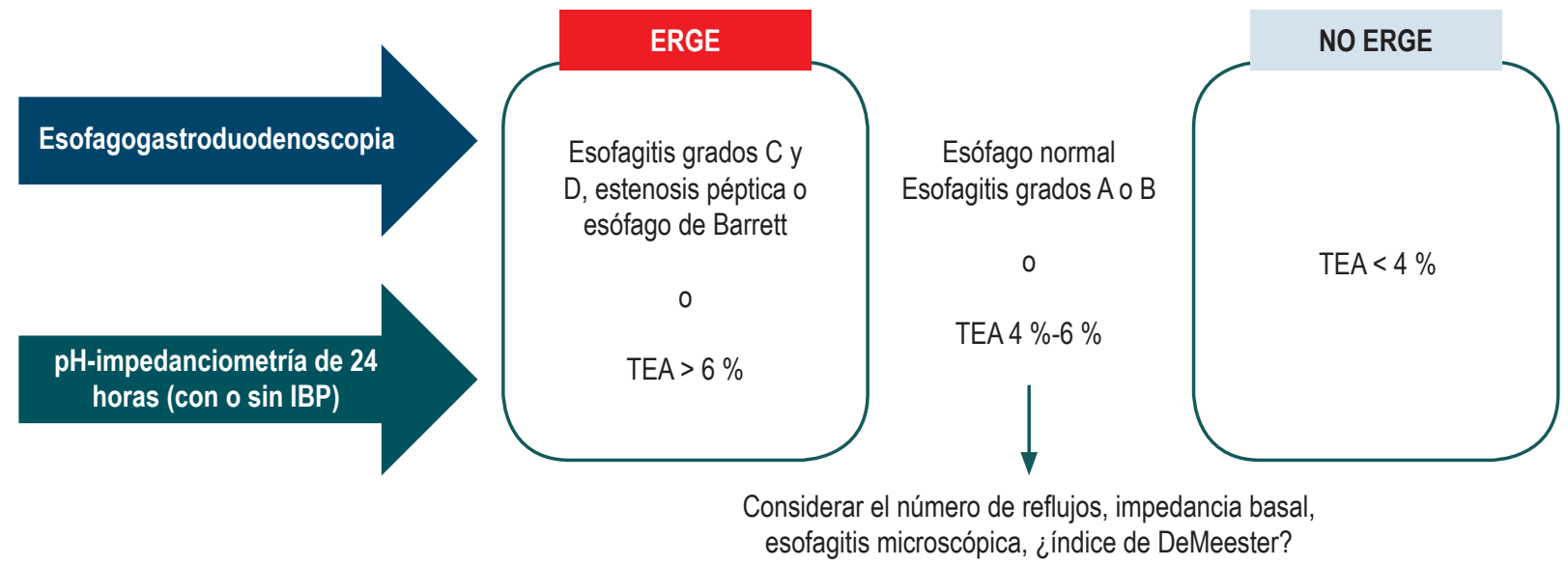

Figura 5. Definición de ERGE. Modificado de: Consenso de Lyon.

se considera positivo cuando es $\geq 50 \%$. Este índice se definió como el número de veces que se presentó el síntoma cuando el $\mathrm{pH}$ es inferior a 4,0, dividido por el número total de veces que se informó el síntoma, multiplicado por el $100 \%$.

La PAS tiene en cuenta el número total de eventos de síntomas, episodios de reflujo y síntomas relacionados con el reflujo; se considera positiva cuando es $>95 \%$. Estas dos evaluaciones son complementarias, miden parámetros diferentes y no se pueden comparar entre sí. Puede ocurrir que el IS sea positivo y la PAS sea negativa y viceversa, situación que representa un área indeterminada y la inter- pretación adicional con otros parámetros es necesaria para definir el diagnóstico; las mediciones que se podrían utilizar son el TEA, número de reflujos e impedancia inicial. La interpretación de estos índices también es útil para determinar diagnósticos diferenciales como pirosis funcional e hipersensibilidad al reflujo. Cuando se tiene una PAS e IS positivos con TEA menor de $4 \%$, se establece el diagnóstico de hipersensibilidad al reflujo.

El diagnóstico de pirosis funcional se define como la presencia de pirosis en un paciente con $\mathrm{pH}$-impedanciometría normal, además de PAS e IS negativos, en quien se han descartado 
otras causas orgánicas que expliquen los sintomas (esofagitis eosinofilica, rumiación y eructo supragástrico) (Figura 6) $(13,15)$.

\section{Paso 5: evaluación del puntaje de DeMeester}

El puntaje de DeMeester se usa para diagnosticar la ERGE desde 1974 con un rendimiento muy similar al TEA y podría tener un papel determinante para definir en casos puntuales cuando un paciente tiene o no ERGE, específicamente en aquellas situaciones en las que los demás parámetros no son definitorios $(5,9)$. En nuestra unidad lo usamos para establecer diagnóstico de ERGE cuando el TEA y el número total de reflujos ácidos son indeterminados. En este caso en particular, un puntaje de DeMeester mayor de 14,7 establece el diagnóstico de ERGE.

\section{Paso 6: impedancia media basal nocturna}

La impedancia media basal nocturna es la expresión de la integridad de la mucosa y proporciona evidencia de daño macroscópico y microscópico esofágico después de la exposición al reflujo. Consiste en medir la impedancia basal 3 a $5 \mathrm{~cm}$ por encima del esfínter esofágico inferior durante el sueño. Se toman mediciones de la impedancia en 3 períodos de 10 minutos para obtener la medida. Los valores menores de 2292 ohmios sugieren la presencia de ERGE y se correlacionaron con pacientes con sospecha de ERGE asociados con síntomas típicos que respondieron al tratamiento con IBP o cirugía antirreflujo $(5,9,16)$.

\section{Paso 7: el índice de onda peristáltica posterior al reflujo inducido por la deglución}

Este índice refleja la integridad del peristaltismo esofágico, estimulado por los episodios de reflujo, y del aclaramiento, debido a la saliva, que contiene una gran cantidad de bicarbonato. Es anormal cuando se presenta una caída anterógrada del $50 \%$ en la impedancia que ocurre dentro de los 30 segundos después de un evento de reflujo que se origina en los sitios de impedancia proximales y alcanza los sitios de impedancia distales, y seguidos al menos de del $50 \%$ de retorno a la línea de base. El valor de corte establecido es del $61 \%(10,16)$.

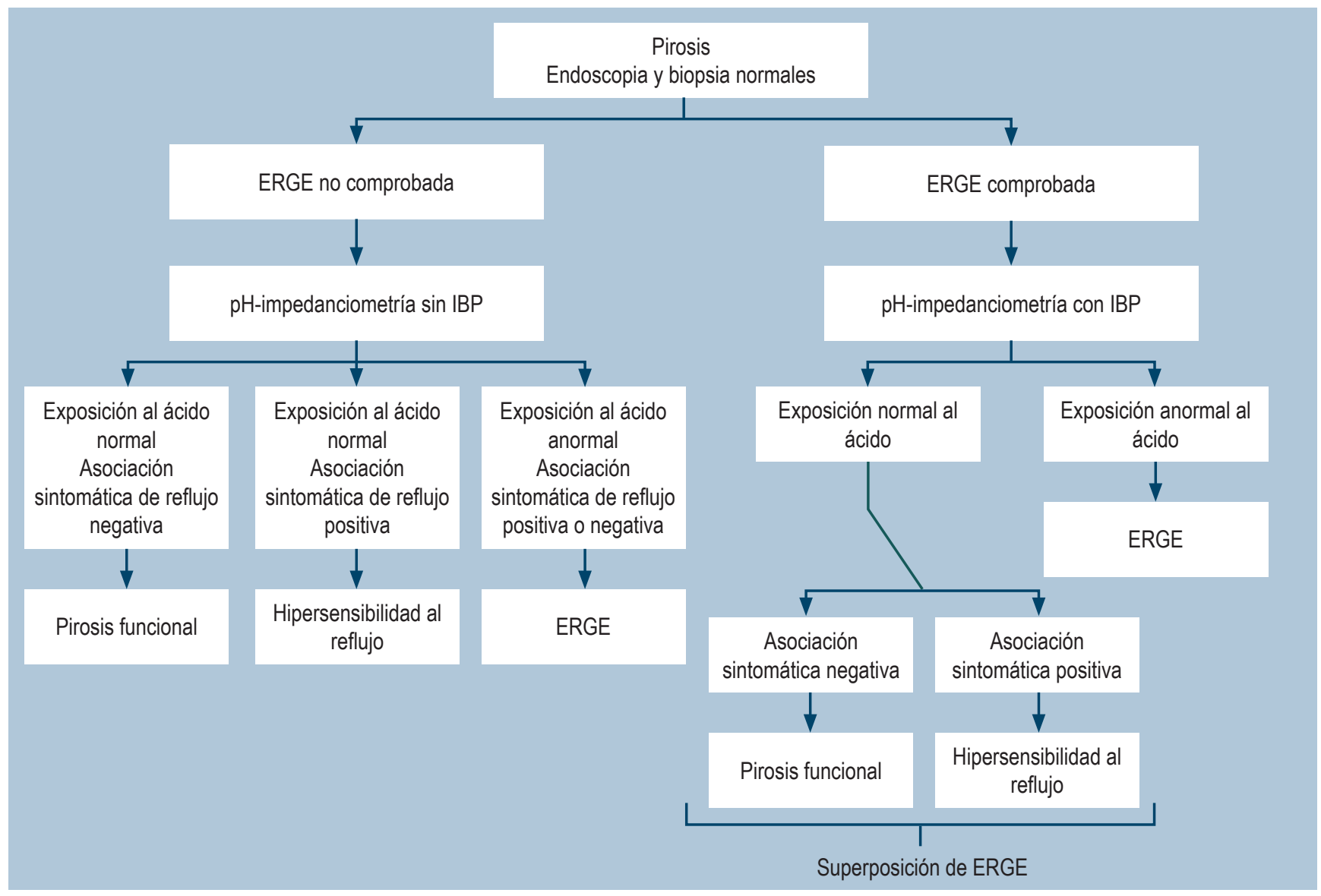

Figura 6. Algoritmo de pirosis funcional e hipersensibilidad esofágica según la clasificación de Roma IV (15). Tomado de: Aziz Qet al. Gastroenterology. 2016;150(6):1368-79. 


\section{Paso 8: evaluar la presencia de rebote ácido nocturno}

La hipersecreción ácida de rebote se define como el aumento de la secreción ácida que se observa después de un período de supresión ácida. Se ha registrado después del tratamiento con bloqueantes de histamina, así como con los IBP. Durante el sueño, la frecuencia de episodios de reflujo disminuye; sin embargo, cuando los pacientes reciben los IBP y están en plan de desprescripción pueden presentar rebote ácido nocturno, el cual se define como un episodio de reflujo ácido de más de una hora de duración en decúbito supino mientras se está dormido (17-20).

\section{CONCLUSIÓN}

El estudio y diagnóstico de la ERGE requiere de la integración de aspectos clínicos, hallazgos endoscópicos y confirmación con documentación objetiva de ERGE con la realización de monitorización de $\mathrm{pH}$-impedanciometría de 24 horas, condiciones que permitirán clasificar a los pacientes en diferentes grupos fenotípicos para poder dirigir en mejor medida sus necesidades terapéuticas. La interpretación por pasos de la $\mathrm{pH}$-impedanciometría permite con un orden lógico poder realizar una lectura adecuada de este estudio de fisiología digestiva, para su posterior aplicación en nuestros pacientes. Es importante informar a los pacientes sobre todas las precisiones técnicas del estudio, con el fin de obtener los resultados más confiables y con la mejor objetivación posible. La pH-impedanciometría a la fecha es el patrón de oro en el diagnóstico de la ERGE con una sensibilidad del $77 \%$ al $100 \%$ y una especificidad del $85 \%$ al $100 \%$.

\section{REFERENCIAS}

1. Vakil N, van Zanten SV, Kahrilas P, Dent J, Jones R; Global Consensus Group. The Montreal definition and classification of gastroesophageal reflux disease: a global evidence-based consensus. Am J Gastroenterol. 2006;101(8):1900-20; quiz 1943. https://doi. org/10.1111/j.1572-0241.2006.00630.x

2. Patti MG. An Evidence-Based Approach to the Treatment of Gastroesophageal Reflux Disease. JAMA Surg. 2016;151(1):73-8. https://doi.org/10.1001/jamasurg.2015.4233

3. Cesario S, Scida S, Miraglia C, Barchi A, Nouvenne A, Leandro G, Meschi T, De’ Angelis GL, Di Mario F. Diagnosis of GERD in typical and atypical manifestations. Acta Biomed. 2018;89(8-S):33-39. https://doi. org/10.23750/abm.v89i8-S.7963

4. Yadlapati R, Pandolfino JE. Personalized Approach in the Work-up and Management of Gastroesophageal Reflux Disease. Gastrointest Endosc Clin N Am. 2020;30(2):227238. https://doi.org/10.1016/j.giec.2019.12.002

5. Gyawali CP, Kahrilas PJ, Savarino E, Zerbib F, Mion F, Smout AJPM, Vaezi M, Sifrim D, Fox MR, Vela MF, Tutuian R, Tack J, Bredenoord AJ, Pandolfino J, Roman S. Modern diagnosis of GERD: the Lyon Consensus. Gut. 2018;67(7):1351-1362. https://doi.org/10.1136/ gutjnl-2017-314722

6. Chen J, Brady P. Gastroesophageal Reflux Disease: Pathophysiology, Diagnosis, and Treatment. Gastroenterol Nurs. 2019;42(1):20-28. https://doi.org/10.1097/ SGA.0000000000000359

7. El-Serag HB, Sweet S, Winchester CC, Dent J. Update on the epidemiology of gastro-oesophageal reflux disease: a systematic review. Gut. 2014;63(6):871-80. https://doi. org/10.1136/gutjnl-2012-304269

8. Páramo-Hernández DB, Albis R, Galiano MT, de Molano B, Rincón R, Pineda-Ovalle LF, Rodríguez A, OteroRegino W, Hani A, Sabbagh LC, Sandoval-Salinas C, Sánchez-Pedraza R. Prevalencia de síntomas del reflujo gastroesofágico y factores asociados: una encuesta poblacional en las principales ciudades de Colombia. Rev Col Gastroenterol. 2016;31(4):337-346.

9. Roman S, Gyawali CP, Savarino E, Yadlapati R, Zerbib F, Wu J, Vela M, Tutuian R, Tatum R, Sifrim D, Keller J, Fox $\mathrm{M}$, Pandolfino JE, Bredenoord AJ; GERD consensus group. Ambulatory reflux monitoring for diagnosis of gastroesophageal reflux disease: Update of the Porto consensus and recommendations from an international consensus group. Neurogastroenterol Motil. 2017;29(10):1-15. https://doi.org/10.1111/nmo.13067

10. Hong SK, Vaezi MF. Gastroesophageal reflux monitoring: $\mathrm{pH}$ (catheter and capsule) and impedance. Gastrointest Endosc Clin N Am. 2009;19(1):1-22, v. https://doi. org/10.1016/j.giec.2008.12.009

11. Hobbs P, Gyawali CP. The role of esophageal $\mathrm{pH}$-impedance testing in clinical practice. Curr Opin Gastroenterol. 2018;34(4):249-257. https://doi.org/10.1097/ MOG.0000000000000441

12. Ribolsi M, Giordano A, Guarino MPL, Tullio A, Cicala M. New classifications of gastroesophageal reflux disease: an improvement for patient management? Expert Rev Gastroenterol Hepatol. 2019;13(8):761-769. https://doi. org/10.1080/17474124.2019.1645596 
13. Neto RML, Herbella FAM, Schlottmann F, Patti MG. Does DeMeester score still define GERD? Dis Esophagus. 2019;32(5):doy118. https://doi.org/10.1093/dote/ doy 118

14. Mainie I, Tutuian R, Shay S, Vela M, Zhang X, Sifrim D, Castell DO. Acid and non-acid reflux in patients with persistent symptoms despite acid suppressive therapy: a multicentre study using combined ambulatory impedancepH monitoring. Gut. 2006;55(10):1398-402. https://doi. org/10.1136/gut.2005.087668

15. Aziz Q Fass R, Gyawali CP, Miwa H, Pandolfino JE, Zerbib F. Esophageal disorders. Gastroenterology. 2016;150(6):1368-79. https://doi.org/10.1053/j.gastro.2016.02.012

16. Savarino V, Marabotto E, Zentilin P, Furnari M, Bodini G, De Maria C, Tolone S, De Bortoli N, Frazzoni M, Savarino E. Pathophysiology, diagnosis, and pharmacological treatment of gastro-esophageal reflux disease. Expert Rev
Clin Pharmacol. 2020;13(4):437-449. https://doi.org/10. $1080 / 17512433.2020 .1752664$

17. Hani de Ardila Albis. Pruebas diagnósticas en enfermedad por reflujo gastroesofágico (ERGE). Rev Col Gastroenterol. 2009;24(2):210-222.

18. Helgadottir H, Bjornsson ES. Problems Associated with Deprescribing of Proton Pump Inhibitors. Int J Mol Sci. 2019;20(21):5469. https://doi.org/10.3390/ ijms20215469

19. Lødrup AB, Reimer C, Bytzer P. Systematic review: symptoms of rebound acid hypersecretion following proton pump inhibitor treatment. Scand J Gastroenterol. 2013;48(5):515-22. https://doi.org/10.3109/00365521.2 012.746395

20. Waldum HL, Qvigstad G, Fossmark R, Kleveland PM, Sandvik AK. Rebound acid hypersecretion from a physiological, pathophysiological and clinical viewpoint. Scand J Gastroenterol. 2010;45(4):389-94. https://doi. org/10.3109/00365520903477348 